\title{
Modeling Deep Neural Networks for Breast Cancer Thermography Classification: A Review Study
}

\author{
Amira Hassan Abed \\ Dept. of Information Systems Center, \\ Egyptian Organization for Standardization \& Quality, Egypt \\ mirahassan61286@gmail.com \\ Essam M Shaaban \\ Dept. of Information Systems, \\ Faculty of computers \& artificial intelligence, \\ Beni-Suef university, Egypt \\ essam.shaban@fcis.bsu.edu.eg
}

\begin{abstract}
Building up a breast cancer screening platform is vital to encourage early "Breast cancer" detection and treatment. Proposing a screening system utilizing clinical imaging methodology that doesn't cause body tissue harm (non-obtrusive) and doesn't include actual touch is a major challenge. Thermography, a "non-intrusive" and "non-contact" malignancy screening strategy, can recognize tumors at the beginning phase significantly under determined conditions by noticing temperature circulation in the two bosoms. The thermograms can be deciphered utilizing Deep learning models, for example, "convolutional neural networks (CNN)". CNNs can naturally group bosom thermograms into classifications, for example, ordinary and up-normal. In this work, we intend to cover the most significant studies identified with the usage of deep neural networks for bosom thermogram classification. As we accept that, an overview of breast thermogram possibilities shows that the early manifestations of bosom malignant can be seen by recognizing the asymmetrical warm dispersions between the bosoms. The asymmetrical warm appropriation on bosom thermograms can be assessed utilizing a computeraided platform that depended on deep learning models.
\end{abstract} Keywords - Breast cancer; convolutional neural networks (CNN); Thermography.

\section{INTRODUCTION}

Worldwide cancer statistics report that "breast cancer" disease is the second most deadly type of cancer after a cellular breakdown in the lungs [1]. In 2018, two billion new breast cancer cases were accounted for around the world and "627,000 deaths". An investigation in Australia [2] indicated that breast cancer endurance is extensively connected with the tumors' size at the period of discovery, with the size under $10 \mathrm{~mm}$, the likelihood of patient endurance is $98 \%$. A companion study indicated that $70 \%$ of bosom disease cases are identified when the cancer size was $30 \mathrm{~mm} \mathrm{[3].} \mathrm{Breast} \mathrm{cancer} \mathrm{generally} \mathrm{gets}$ discernible during screening when the tumor is more than $20 \mathrm{~mm}$ in size [4]. Hence, empowering early identification of cancer disease is critical to encourage early treatment. Early treatment might be useful after detection by screening assessments, for example, "Clinical- breast examination (CBE)" and "breast self-examination (BSE)". $\mathrm{CBE}$ is a standard clinical assessment performed by medical services experts to distinguish bosom injuries, while BSE is tested by a person to notice actual changes and the presence of bosoms. The act of BSE engages ladies to assume liability for their wellbeing. Thus, BSE is suggested by the "World Health Organization" for bringing awareness among ladies [5].
Screening strategies generate clinical images of bosoms. The understandings of these images are regularly done by specialists. Several studies mentioned that low thermograms analytic precision resulted from weak specialists' capabilities and skills in deciphering such images. The development of different illnesses and restricted human capabilities has propelled analysts and medical experts to utilize computer-aided innovation to encourage breast thermography-based analysis and accordingly limit blunders. Thusly, a computer-assisted framework is required to characterize thermograms automatically into typical and abnormal classes. Thinking about this necessity, many studies toward proposing a computer-assisted system for examining clinical images has been consistently increasing. Numerous PC-aided strategies for the examination have been implemented to help medical teams deciphering the clinical images. Over the last few years, critical exertion has concentrated on the improvement of deep learning (DL) models. Since DL models are openly accessible, they can be employed effectively utilizing pre-trained networks. In breast cancer identification, numerous existing studies depend on DL utilizing mammograms [6, 7, 8], histology pictures [9], tomosyntheses [10], and ultrasound pictures [11] have demonstrated palatable accuracies.

However, scarcely some investigations have added to non-obtrusive thermal of bosoms utilizing the DNN 
procedure. Considering existing restricted assets, the research on this issue is still at its beginning phases. Thus critical exertion is needed to create a dependable computer-aided system to empower the early recognition of breast disease. This requires an investigation of significant past, new and vital future exploration studies on thermal imaging and deep learning for bosom malignant identification ought to be considered of foremost significance. Through our work, we survey current advancements in breast disease identification utilizing deep learning and thermography as a non-obtrusive methodology. This work is coordinated as follows. Section 2 presents the Breast cancer Background. Section 3 defines thermography and explains its role in Breast cancer.

\section{BREAST CANCER BACKGROUND}

Breast cancer is reported as the most considerably announced cancer type in the entire world. This kind of cancer has been accounted for between males and females; in any case, its recurrence with females is high in comparison to males' cases. This is straightforwardly considered based on the fundamental difference between the breast in the two sexes; where the successive screening of dangerous cells are found in milk-making centers, lobules, and milk moving trenches, channels. Figure 1 shows an anatomical perspective on the female bosom with the repeatedly common areas of breast malignancy series. The advancement of breast cancer may happen because of obscure reasons. In any case, one of the affirmed bosom malignancy activities is the strange improvement of the living cells. There are specific genes that are responsible for the split and increase of cells, yet for certain reasons, these genes fizzle in identifying the irregularities.

This prompts a fast development and propagation of dead cells that have not to be split and increased; generating a type of tumor. The generated tumor is classed as harmful in the case of the prevalence over and assaults its encompassing; when it stays involved in specific tissues, similar to conduits and lobules, it is ordered as a non-intrusive tumor. It is critical to invoke that cancers' cells would spread to any part of the human body; in breast malignancy, they move across the "lymph" or "blood". In the last situation, the bosom disease is considered in its high-level stage and surgical mediation, called "biopsy", is typically requested.

It is enthusiastically prescribed that patients have bosom cancer before it converts to this high-level stage. For this particular explanation, breast cancer screening techniques and devices are developed for detection purposes following the standard methodology. These techniques differ in their procedures, applications, and results, yet there is no preferred technique for physicians.

Frequently, doctors require screening using various techniques to affirm the acquired outcomes. In any case, a point that can be considered is the side effect of the screening technique. The new headways in the automated operation have animated scientists to re-investigate screening techniques for upgrade purposes. These days, it is very conceivable to discover a reexamination of beforehand non-proficient breast screening strategies and adjusting them to the accessible handling and clustering technologies to deliver extensive outcomes.

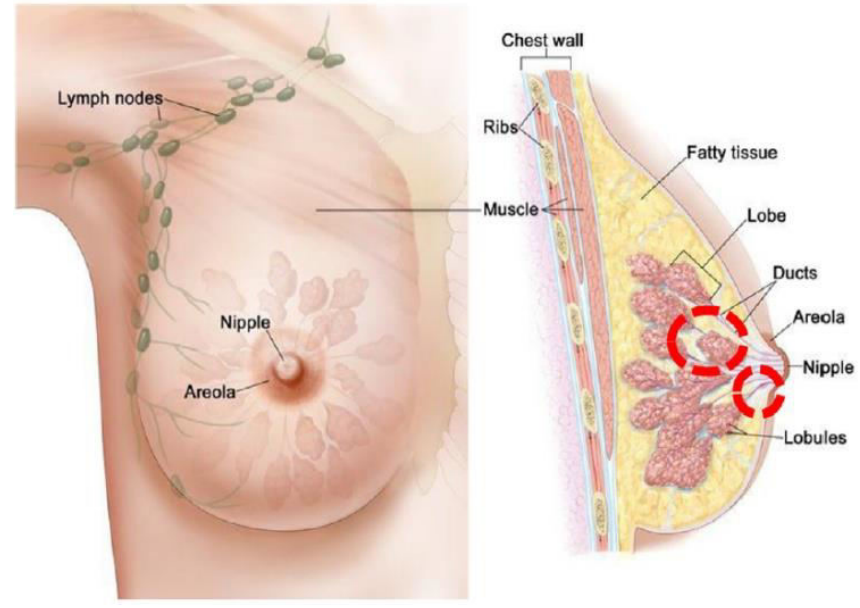

Fig. 1: Common cancer incidence locations in the female breast.

\section{THERMOGRAPHY}

Thermography is a prescient method for observing the state of plant hardware constructions and frameworks, not simply electrical gear. It utilizes instrumentation to peruse infrared energy outflows (surface temperature) to decide working conditions. Infrared thermography (IRT), "thermal imaging", and "thermal video" are instances of the infrared imaging field. Thermographic cameras as a tool for distinguishing radiation in the long-infrared scope of the electromagnetic range ("approximately 9,000 14,000 nanometers or 9-14 $\mu \mathrm{m}$ ") and generate pictures of that radiation, called "thermograms". As infrared radiation is produced by any object that owns a temperature above outright zero based on the dark body radiation law, thermography enables conceivable to see human current circumstance with or without obvious illumination.

The measure of radiation produced by any entity increments with temperature; thusly, thermography permits us to see assortment in temperature. During screening using thermal imaging cameras, thermal entities stand apart well against cooler foundations; people and other warm-blooded creatures become effectively noticeable against the climate, day or night. Subsequently, thermography is especially helpful to the military and different users of reconnaissance cameras.

\section{THERMOGRAPHY IN THE DIAGNOSIS OF BREAST CANCER}

Thermography has a progression of novel facilities. One of these facilities is the capability for early discovering of cancer by recognizing early indications of disease, ten years sooner than different techniques, for example, mammography [12]. Another novel capability of thermography is assessment procedures for malignancy treatment. Anticipate the future of the patient state is likewise one of the thermography facilities [13]. It has been indicated that $44 \%$ of patients with anomalous 
thermograms, around five years after thermography, have been determined to have breast cancer.

Thermography likewise indicated that there is a $24 \%$ likelihood of survival in 3 years after determining that a specific patient has a high level of malignancy, where this likelihood for tumors at prior levels is near $80 \%$ [13]. The affectability of mammography in more youthful individuals or females with thick bosom tissue will be diminished. Yet, thermography is the freedom to the patients' ages and the thickness of bosom cancers. In $70 \%$ of patients, thermography can recognize manifestations of breast cancer one year sooner than mammography [13]. The size of imperceptible tumors by mammography is about $1.66 \mathrm{~cm}$ while restricting size in thermography is abatement to $1.28 \mathrm{~cm}$. As a finder for breast cancer risk in the future, an anomalous thermogram in contrast with an ordinary thermogram can exhibit the danger of breast cancer "22 times". Additionally, the irregular thermogram is "10 times" more significant and compelling than the first family member's history of the illness. Thermal pictures examination can be gathered by "Thermobiology" in Five primary classes: TH1- "normal uniform non-vascular", TH2- "vascular ordinary uniform", TH3- "vague (questionable)", TH4- "abnormal", TH5-“extremely abnormal", example classified pictures are appeared in Figure 2 [14].

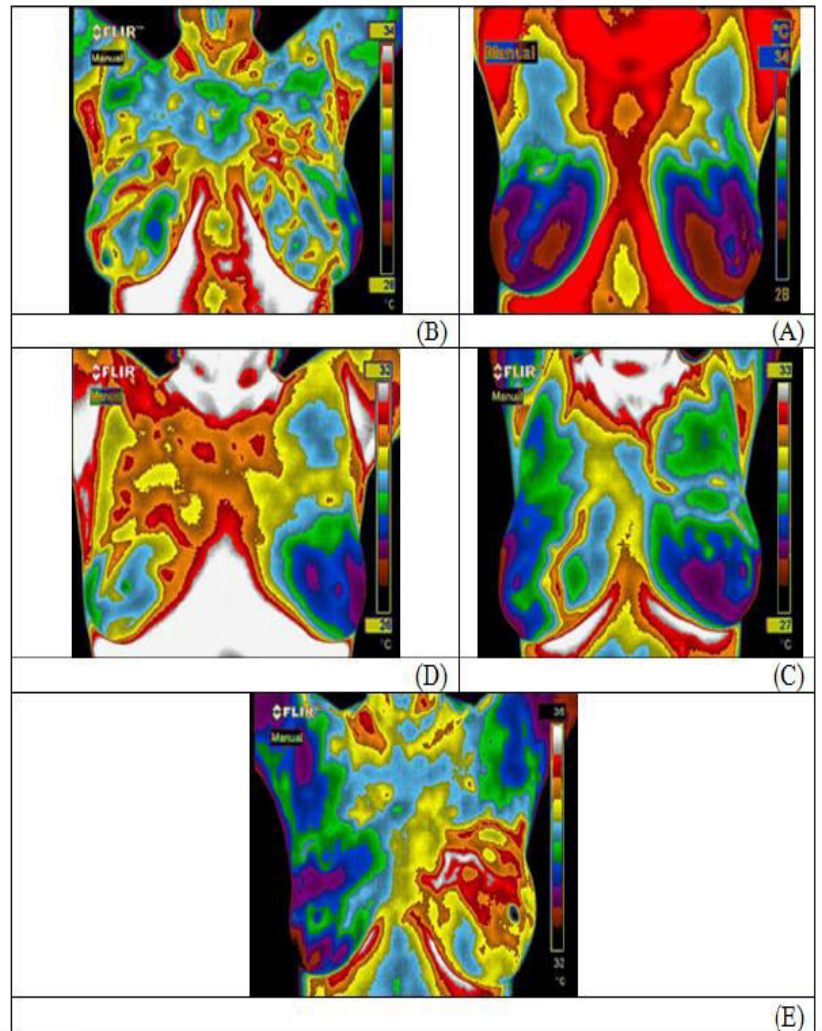

Figure 2: Thermal images classification based on: (A) TH1 - normal

uniform non-vascular, (B) TH2- vascular ordinary uniform, (C) TH3-

vague (questionable), (D) TH4- abnormal, (E) TH5extremely abnormal [14].

\section{DEEP LEARNING FOR BREAST THERMOGRAM CLASSIFICATION}

The neural networks NNs are roused by how neurons in the human cerebrum process. Every human brain's neuron is interconnected and information streams through every neuron. In NNs, neurons get input and play out many activities with "weights" and "biases". Weight indicates the strength of the association between two hubs. Biases are outside values that increment or decline the net input of the activation work. Hubs are the individual preparing units in each layer. Figure 3 outlines the mathematical model of a neuron.

There are two primary objectives of Learning from data: to comprehend the data streaming process and its interpretation; and to foresee future perceptions. Foreseeing future solutions doesn't need a probabilistic accuracy rate. Be that as it may, accuracy is a focal point of clinical information interpretations. As applied in identifying breast cancer, $100 \%$ precision is needed to guarantee that the determination follows the strong ground. $\mathrm{NN}$ is a huge parallel circulated processor comprised of straightforward processing units, which has a characteristic penchant for solid knowledge, making it accessible for utilizing. The NN algorithm permits the learning of the subjective values of medical images. Consequently, it is proper for interpretations of bosom thermogram characterization.

A "convolution neural organization" $(\mathrm{CNN})$ is a deep neural network technique in which it processing the input pictures by allotting certain learnable "weights and biases" to locate significant features which separate one picture from others. Thusly, the classification output can be seen as the yield. Figure 4 supports the overall CNNs building for the classification of the bosom thermograms in two sets, normal and cancer. Three significant contemplations should be focused on: "dataset readiness in picture prepreparing", "feature learning", and "classification". The classification can be double ("normal and cancer"), or many classes, for example, solid, generous, and harmful. In the accompanying sections, we survey the ideas and related endeavors in $\mathrm{CNN}$ usage for classification breast thermogram.

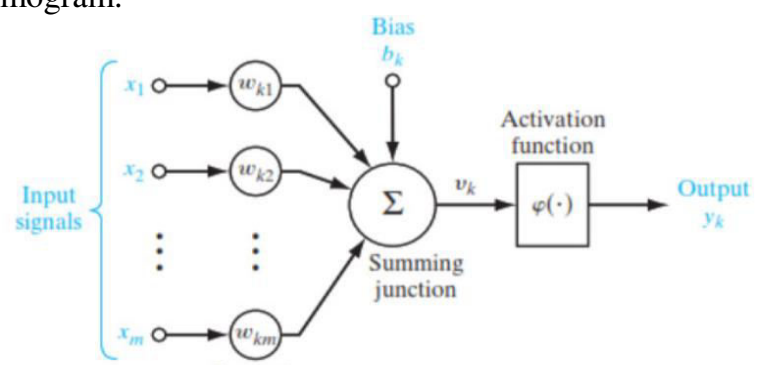

Figure 3: Neural Network resembles the human nervous system.

\section{A. Preprocessing Images process}

Preprocess of Images is a significant process to enhance the features of images, by stifling undesirable information and upgrading significant features of images to improve the NN algorithm performance. Preprocessing 
Images process is vital for NNs as the accomplishment of the learning interaction relies upon the features gaining from input Images. For the most part, Preprocessing of Images incorporates mean deduction, standardization, PCA brightening, and neighborhood contrast standardization.

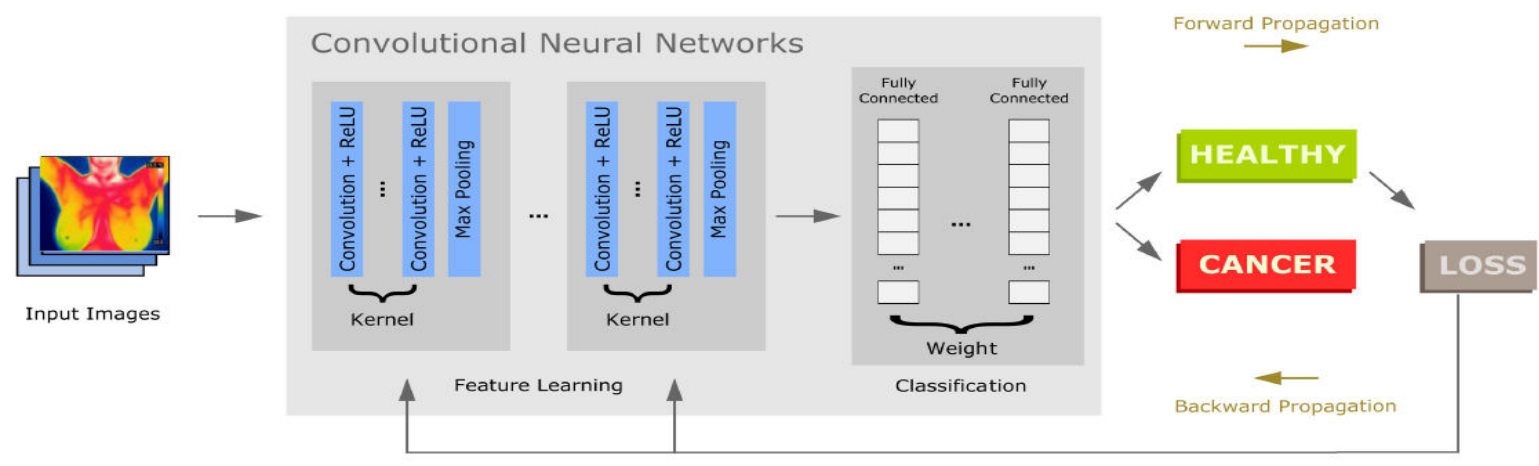

FIGURE 4: Convolutional Neural Networks for BC early detection using breast thermograms

Furthermore, many algorithms are utilized for bosom thermograms pre-preparing incorporate resizing, segmentation of the "region of interest (ROI)", and expansion.

\section{B. Convolutional Neural Networks (CNNs)}

NNs are ordinarily utilized for perceiving and identifying objects in picture input data. All in all, CNN is like other NNs, having weight, predisposition, and the initiation work when preparing inputs. Be that as it may, $\mathrm{CNN}$ empowers features extraction to define patterns from high-dimensional sources of data. This interaction is named "convolution" and is implemented in a "convolutional layer" (feature extraction layer). As demonstrated in Figure 6, CNN has two significant layers: "feature extraction" and "fully connected networks" that were clarified in the accompanying subsections.

\section{1) Feature Extraction Layer}

The feature extraction layer is responsible for encoding to produce features of input data (images). so, one picture as input data is encoded to be a "feature map" containing numbers that define picture "characters". This layer includes two implementation parts: "convolution" and "pooling". The "convolution" part is organized as a "filter" (kernel) with a specific size is shaped. Given that the bosom thermogram contains three shading channels (RGB), there are three filters as per the channels. Thus, the yield of the convolution layer is a "feature map" that is then sent to the "pooling layer". The pooling layer contains one channel with a specific size of the step. In the convolutional layer, include maps are up-tested, though, in the pooling layer, feature maps are down-examined. There are two ordinarily kinds of pooling initiation works that are typically being utilized: "max pooling" and "average pooling". The greatest estimation of the feature maps is chosen in max-pooling, but the average estimation of all feature maps is chosen in average pooling.

In the "learning features", CNNs repeat the processes of convolution and max-pooling until perceive the "features" of the images. Figure $\mathbf{5}$ delineates the convolution cycle in $\mathrm{CNN}$ utilizing bosom thermograms as the inputs pictures. As the inputs have three channels (RGB), the kernel volume additionally involves three individual "2D kernels". Each channel correlated with one kernel. The size of the kernel is resolved by the number of feature maps. of feature maps.

\section{2) Fully-Connected Layer}

Feature maps obtained because of the "convolutional layer" are as a multi-level array. Along these lines, it is important to level or reform the feature map into a onedimensional array (vector) before it is utilized as the contribution for the completely associated layer. As such, completely associated layers relate to convolutional layers with a filter size of $1_{-}{ }_{1}$. A completely associated layer is otherwise called a thick layer, in which each input is associated with every output based on learnable weights. At the point when features are determined by convolutional layers and down-examined by pooling layers, they are planned by a subset of completely associated layers to the last yields of the network, for example, probabilities of every class in classification undertakings. The last completely associated layer ordinarily has a similar number of resulted hubs as classes [16].

\section{Images Classification}

Classifying Images is the way toward grouping pictures as indicated by their visual substance. The "training process" for NNs includes perceiving thermograms of breasts with identified labels e.g., normal and up-normal. This test is called "supervised learning" [17]. During the Classification of an item into various classes, CNNs regularly settle on a decision from a probabilistic perspective, which is named "inference". This implies that yield probabilities will be an array of numbers somewhere in the range of 0 and 1 . One basic kind of the resulted model is the "softmax function" that ascertains the likelihood appropriation of an event. In CNNs, the "softmax function" computes the likelihood of a resulted picture over the conceivable target classes. 


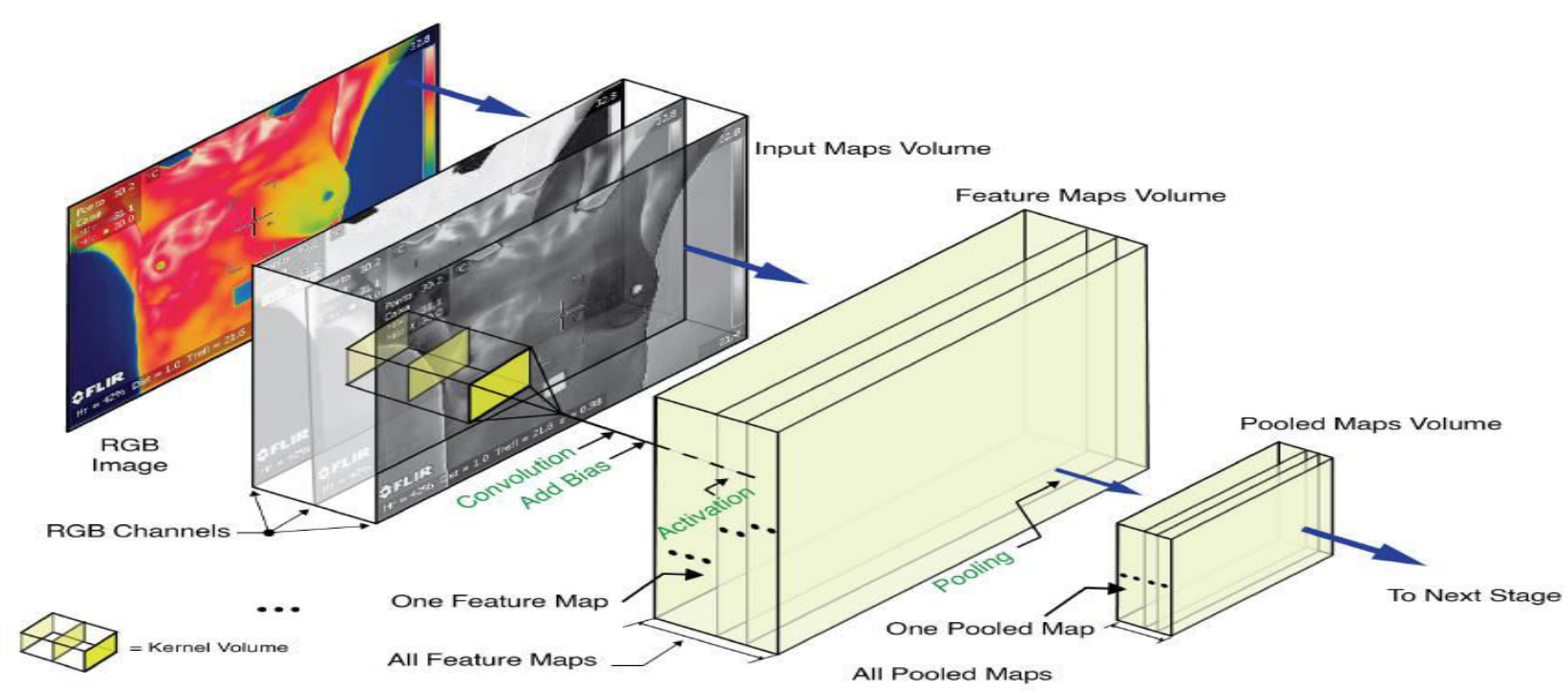

Figure 5: Visualization of the convolutional process of a cancerous breast thermogram; modified from a previous study [15].

\section{REVIEW ON BREAST CANCER USING THERMOGRAPHS AND DEEP LEARNING}

Recently as shown in table $\mathbf{1}$, in 2020, Samir S. \& Shivajirao M. [18] introduced an impressive proof in which thermography is a significant and disparaged technique. They have utilized a novel machine learning approach (CNN) over conventional AI methods to characterize bosom disease utilizing warm pictures. They have utilized "two pre-trained transfer learning models"; "VGG16" and "InceptionV3" to optimize the execution of their gauge CNN model. The best outcome was obtained from the "InceptionV3" model. In the same year, Sami E. \& Hushang J. [19] created programming for early identifying bosom malignancy consequently which utilizations picture preparing methods and calculations to break down thermal breast pictures to distinguish the indications of the sickness in these pictures. Utilizing this new technique the features will be separated from the warm pictures caught using thermal cameras and could be utilized to group the breast pictures as ordinary or upnormal through utilizing "convolutional neural organizations (CNNs)" streamlined based on the Bayes algorithm. In another investigation by Hossein Gh. et al. [20] introduced a new strategy dependent on PC-supported diagnosis innovation for the early discovery of sickness in patients with asymptomatic breast malignancy. Since in thermography, the areas with higher temperatures are inclined to physiological issues, the designed method depends on the factual examples of the worm districts of the breast. Along these lines, they attempted to distinguish irregular warmth patterns by introducing fitting features in thermal pictures depended on a progression of people's pictures and "Deep Neural Network" methods for enhancing the precision of finding variations in bosom cancer.

In August 2019, Francisco et al. [21] introduced a complete report for the early discovery of bosom cancer. They investigated the prescient exactness of various CNN designs: "resnet18", "resnet34", "vgg16" and "vgg19", to group patients with bosom cancer versus normal utilizing "tomographies". They have indicated that "Resnet" lingering models show amazing outcomes in the arrangement of carcinoma from thermographies, particularly "Resnet18", "Resnet34", and "Resnet50". Furthermore, expanding the number of layers doesn't optimize the approval exactness, when contrasted with the outcomes got for "Resnet152". "Resnet" structures show a better presentation than "Vgg" models. In the next month Sebastien M. [22], introduced a Novel procedure that relied on an "inceptionV3 couples k-Nearest Neighbors (InceptionV3-KNN)" and a specific module that they named: "StageCancer." These methods prevail to order breast cancer in 4 phases (T1: non-obtrusive bosom disease, T2: the tumor matches $2 \mathrm{~cm}, \mathrm{~T} 3$ : the tumor is bigger than $5 \mathrm{~cm}$ and T4: the full bosom is cover by malignant growth). They detailed that it is the first occasion when that such classification is finished utilizing warm pictures of the bosoms. Their outcomes guarantee that infrared imaging combined with a ground-breaking PC Help Gadget (computer-aided design) can prompt an exact tumor finder.

In the following month, Juan Carlos et al. [23], propose a computerized examination way to deal with survey the abilities of deep neural networks for bosom thermograms classifications. All pictures were ordered with seven diverse profound learning pre-prepared structures. The "deep neural networks VGG-16" demonstrated the best execution, accomplishing the Best Acc of 91.18\%. The outcomes recommend that bosom disease thermography related to deep neural organizations can be utilized as subordinate to mammography for pre-screening, even though there are still bogus positives. Information growth has been appeared to expand the exactness of deep neural organizations with a restricted dataset, for example, for their situation. 
TABLE 1: Review on breast cancer thermography using deep learning

\begin{tabular}{|c|c|c|c|c|c|c|c|}
\hline References & Task & Technique & $\begin{array}{c}\text { Acquisition } \\
\text { Protocol } \\
\end{array}$ & $\begin{array}{c}\text { Image } \\
\text { Dimensions }\end{array}$ & Dataset & Accuracy & CNN Models \\
\hline$[18]$ & Classification & $\mathrm{CNN}$ & $\begin{array}{l}\text { Static \& } \\
\text { Dynamic }\end{array}$ & $\begin{array}{c}\text { 3-D } \\
\text { image }\end{array}$ & $\begin{array}{l}1140 \text { image of } \\
\text { "DMR-IR } \\
\text { dataset" }\end{array}$ & $93.1 \%$ & $\begin{array}{l}\text { "VGG16, Baseline, } \\
\text { Inception-V3" }\end{array}$ \\
\hline [19] & Classification & $\mathrm{CNN}$ & $\begin{array}{l}\text { Dynamic } \\
\text { protocol }\end{array}$ & $\begin{array}{l}\text { 2- D } \\
\text { image }\end{array}$ & $\begin{array}{l}3895 \text { image of } \\
\text { "visual lab } \\
\text { dataset" }\end{array}$ & $96.7 \%$ & "Bayes optimization" \\
\hline$[28]$ & Classification & $\mathrm{CNN}$ & $\begin{array}{l}\text { Dynamic } \\
\text { protocol }\end{array}$ & $\begin{array}{l}2-\mathrm{D} \\
\text { image }\end{array}$ & $\begin{array}{l}680 \text { image of } \\
\text { "visual lab } \\
\text { dataset" }\end{array}$ & $95.8 \%$ & "ReLU" \\
\hline [29] & Classification & $\mathrm{CNN}$ & $\begin{array}{l}\text { Static \& } \\
\text { Dynamic }\end{array}$ & $\begin{array}{l}\text { 3-D } \\
\text { image }\end{array}$ & $\begin{array}{l}1140 \text { image of } \\
\text { "DMR-IR } \\
\text { dataset" }\end{array}$ & $91.32 \%$ & "TensorFlow" \\
\hline [20] & Classification & DNN & $\begin{array}{l}\text { Dynamic } \\
\text { protocol }\end{array}$ & $\begin{array}{c}2-D \\
\text { image }\end{array}$ & $\begin{array}{c}1,960 \text { image } \\
\text { from "Vision } \\
\text { Lab" } \\
\end{array}$ & $96.77 \%$ & "Sparse" \\
\hline [22] & Classification & DNN & $\begin{array}{l}\text { Dynamic } \\
\text { protocol }\end{array}$ & $\begin{array}{l}\text { 2- D } \\
\text { image }\end{array}$ & $\begin{array}{c}1062 \text { image } \\
\text { from "Vision } \\
\text { Lab" }\end{array}$ & $91.8 \%$ & "InceptionV3-KNN" \\
\hline [21] & Classification & $\mathrm{CNN}$ & $\begin{array}{l}\text { Dynamic } \\
\text { protocol }\end{array}$ & $\begin{array}{c}\text { 3- D } \\
\text { image }\end{array}$ & $\begin{array}{c}2.411 \text { image } \\
\text { from "Vision } \\
\text { Lab" }\end{array}$ & $96 \%$ & $\begin{array}{l}\text { "ResNet 18, ResNet 34, } \\
\text { ResNet 50, ResNet 152, } \\
\text { VGG16 \& VGG19" }\end{array}$ \\
\hline$[25]$ & Classification & $\mathrm{CNN}$ & $\begin{array}{l}\text { Dynamic } \\
\text { protocol }\end{array}$ & $\begin{array}{l}\text { 3- D } \\
\text { image }\end{array}$ & $\begin{array}{l}7800 \text { image } \\
\text { from "Vision } \\
\text { Lab" }\end{array}$ & $91.8 \%$ & "VGG16 \& VGG19" \\
\hline [30] & Classification & $\mathrm{CNN}$ & $\begin{array}{c}\text { Static } \\
\text { protocol }\end{array}$ & $\begin{array}{c}\text { 3- D } \\
\text { image }\end{array}$ & $\begin{array}{l}37 \text { image of } \\
\text { "DMR-IR } \\
\text { dataset" }\end{array}$ & $93.42 \%$ & "SMO classifier" \\
\hline [31] & Classification & $\mathrm{CNN}$ & $\begin{array}{c}\text { Static \& } \\
\text { Dynamic }\end{array}$ & $\begin{array}{c}\text { 3- D } \\
\text { image }\end{array}$ & $\begin{array}{l}1140 \text { image of } \\
\text { "DMR-IR } \\
\text { dataset" }\end{array}$ & $94 \%$ & $\begin{array}{c}\text { "ResNet101, DenseNet, } \\
\text { MobileNetV2 \& } \\
\text { ShuffleNetV2" }\end{array}$ \\
\hline$[23]$ & Classification & $\mathrm{CNN}$ & $\begin{array}{c}\text { Static } \\
\text { protocol }\end{array}$ & $\begin{array}{c}\text { 2-D } \\
\text { image }\end{array}$ & $\begin{array}{l}173 \text { image of } \\
\text { "DMR-IR } \\
\text { dataset" }\end{array}$ & $91.18 \%$ & $\begin{array}{l}\text { "AlexNet, GoogLeNet, } \\
\text { ResNet-50, ResNet-101, } \\
\text { InceptionV3, VGG-16 } \\
\text { \& VGG-19" }\end{array}$ \\
\hline [24] & Classification & $\mathrm{CNN}$ & $\begin{array}{l}\text { Dynamic } \\
\text { protocol }\end{array}$ & $\begin{array}{c}\text { 2-D } \\
\text { image }\end{array}$ & $\begin{array}{l}1140 \text { image of } \\
\text { DMR-IR } \\
\text { dataset }\end{array}$ & $92 \%$ & $\begin{array}{l}\text { "ResNet, SeResNet, } \\
\text { VGG16, Inception, } \\
\text { InceptionResNetV2 and } \\
\text { Xception" }\end{array}$ \\
\hline [26] & Classification & $\mathrm{CNN}$ & $\begin{array}{c}\text { static \& } \\
\text { dynamic }\end{array}$ & $\begin{array}{c}2-\mathrm{D} \\
\text { image }\end{array}$ & $\begin{array}{l}1017 \text { image of } \\
\text { "DMR-IR } \\
\text { dataset" }\end{array}$ & $95 \%$ & $\begin{array}{c}\text { "ResNet 50, VGG16 \& } \\
\text { VGG19" }\end{array}$ \\
\hline [27] & Classification & $\mathrm{CNN}$ & $\begin{array}{l}\text { Dynamic } \\
\text { protocol }\end{array}$ & $\begin{array}{c}\text { 2-D } \\
\text { image }\end{array}$ & $\begin{array}{c}750 \text { image of } \\
\text { visual lab } \\
\text { dataset }\end{array}$ & $92.1 \%$ & $\begin{array}{c}\text { "U-Net, V-Net, } \\
\text { VGGNet \& } \\
\text { InputCascade CNN" }\end{array}$ \\
\hline
\end{tabular}

In October 2019, J. Zuluaga-Gomez et al. [24], introduced a CNN-based technique for bosom disease analysis utilizing warm pictures. They indicated that an allaround delimited data set split strategy is required to diminish the inclination and over-fitting through the preparation stage, in this manner their exploratory outcomes affirm that. Furthermore, this paper passes on the primary cutting edge benchmark of $\mathrm{CNN}$ models, for example, "ResNet", "SeResNet", "VGG16", "Inception ", "Inception ResNetV2" and "Xception" for the DMR-IR data set. Similarly, this investigation builds up the primary CNN hyper-boundaries streamlining in a thermography data set for bosom disease, where the top CNN model accomplished $92 \%$ exactness. They showed that the compromise between database size and data enlargement procedures is critical in classification assignments lacking adequate information.

Toward the end of the year, Hafiz T. et. al. [25], introduced the framework of a "thermography based application-explicit Advanced Back End (DBE)" processor 
for a shrewd screening tool. Thermal pictures of the chest taken by infrared cameras are pre-prepared to specify the districts of interest. To accomplish productive equipment features texture features are deliberately chosen, which are then taken off to a double classifier depended on trained "Linear Support Vector Machine" and "CNN" to choose the decision boundary. The presented framework accomplishes proficient equipment usage by misusing developed classifiers. In 2018, Matheus \& Lucas [26], build a supervised strategy for dissecting infrared thermography of bosom for exact classification utilizing CNN that not depends on hand-tailored features. They propose 4 procedures to decide how the unique convention fits better in a CNN algorithm. The outcomes demonstrated that their DL approach utilizing the shaded picture dataset gave great execution, not as a grayscale dataset for "static protocol" and those CNNs got serious outcomes for the two conventions: "static" and "dynamic". They expect that since substantially more data about temperature is put away in the shading set and CNN features catch patterns is a more productive way than hand-tailored feature selection. Following this investigation, Aman D. [27] indicated that the division of problem areas in a thermal picture is an intense issue generally because of the un-accessibility of huge bosom thermography datasets, absence of standardized information, and the reliance of caught warm pictures on mood, enthusiastic and actual condition of the subject. In this study, they investigate different CNN models for "semantic segmentation" beginning from "naive patch" based classifiers to more refined ones including a few varieties of the encoder-decoder network for recognizing the areas of interest in the warm pictures. The results investigate the importance of the utilization of multi-layered $\mathrm{CNN}$ for the identification of areas of interest in infrared bosom warm pictures.

\section{CONCLUSION}

Early breast cancer recognition stays the foundation of bosom disease control. Bosom self-assessment is prescribed by the "World Health Organization" to raise awareness of ladies about bosom malignancy hazards. Thermography is designed for performing an early localization via screening technique, and we accept that it gives a promising improvement for a self-screening strategy that would identify bosom malignancy at a beginning phase. An outline of the breast thermogram may demonstrate that the early indications of bosom cancer can be seen through distinguishing the lopsided thermal conveyances between the bosoms.

The lopsided thermal distribution on bosom thermograms would be assessed utilizing PC -aided innovation. The utilization of this innovation can limit mistakes. Our review had demonstrated that the present NN models have prompted an optimization in the classification exactness of breast cancer thermogram, especially in distinguishing among normal and harmful cases. Nevertheless, the NNs model performance should be improved. Future research requires more effort for improving bosom thermograms classification. This will need giving delegate datasets, getting ready great ROIs, allocating great kernels, executing "lightweight" CNN models. The accomplishment of these goals will abbreviate the time related to convolution calculation and increment precision rates. A free detection technique utilizing thermography can then be built for a self-bosom detection tool at a beginning phase without requiring actual inclusion.

\section{REFERENCES}

1. IAFR Cancer. Global Cancer Observatory. Accessed: Feb. 3, 2021. [Online]. Available: http://gco.iarc.fr/

2. C. Nickson and A. M. Kavanagh, "Tumour size at detection according to different measures of mammographic breast density," J. Med. Screening, vol. 16, no. 3, pp. 140_146, Sep. 2009.

3. S. A. Narod, "Tumour size predicts long-term survival among women with lymph node-positive breast cancer," Current Oncol., vol. 19, no. 5, pp. 249_253, Sep. 2012.

4. OncoLink Team. (Jan. 2021). All About Breast Cancer. [Online]. Available: https://www.oncolink.org/cancers/breast/all-aboutbreast-cancer

5. Breast Cancer: Prevention and Control, World Health Org., Geneva, Switzerland, 2019.

6. H. Chougrad, H. Zouaki, and O. Alheyane, "Deep convolutional neural networks for breast cancer screening," Comput. Methods Programs Biomed., vol. 157, pp. 19_30, Apr. 2018.

7. M. A. Al-Masni, M. A. Al-Antari, J.-M. Park, G. Gi, T.-Y. Kim, P. Rivera, E. Valarezo, M.-T. Choi, S.M. Han, and T.-S. Kim, "Simultaneous detection and classification of breast masses in digital mammograms via a deep learning YOLO-based CAD system," Comput. Methods Programs Biomed., vol. 157, pp. 85_94, Apr. 2018.

8. J. Arevalo, F. A. González, R. Ramos-Pollán, J. L. Oliveira, and M. A. G. Lopez, "Representation learning for mammography mass lesion classification with convolutional neural networks," Comput. Methods Programs Biomed., vol. 127, pp. 248_257, Apr. 2016

9. D. Bardou, K. Zhang, and S. M. Ahmad, "Classification of breast cancer based on histology images using convolutional neural networks," IEEE Access, vol. 6, pp. 24680_24693, 2018.

10. X. Zhou, T. Kano, H. Koyasu, S. Li, T. Hara, X. Zhou, M. Matsuo, and H. Fujita, "Automated assessment of breast tissue density in non-contrast 3D CT images without image segmentation based on a deep CNN," Proc. SPIE, vol. 10134, Mar. 2017, Art. no. 101342Q.

11. H. Li, J. Weng, Y. Shi, W. Gu, Y. Mao, Y. Wang, W. Liu, and J. Zhang, “An improved deep learning approach for detection of thyroid papillary cancer in ultrasound images," Sci. Rep., vol. 8, no. 1, pp. 1_12, Dec. 2018.

12. Amira H. Abed, M. Nasr \& W. Saber "The Future of Internet of Things for Anomalies Detection using 
Thermography", International Journal of Advanced Networking and Applications (IJANA), Volume 11 Issue 1, pp. Pages: 4142-4149 (2019).

13. G. Ahmad, Z. Iman, G. Hossein \& H. Javad "A review of the dedicated studies to breast cancer diagnosis by thermal imaging in the fields of medical and artificial intelligence sciences," Biomedical Research 2016; 27 (2): 543-552

14. Thermobiological 2016. Available at: http://www.breastthermography.com/breast_ther mography_proc.htm.

15. R. C. Gonzalez, "Deep convolutional neural networks [lecture notes]," IEEE Signal Process. Mag., vol. 35, no. 6, pp. 79_87, Nov. 2018.

16. R. Yamashita, M. Nishio, R. K. G. Do, and K. Togashi, "Convolutional neural networks: An overview and application in radiology," Insights Image, vol. 9, no. 4, pp. 611_629, Aug. 2018.

17. O. Simeone, "A brief introduction to machine learning for engineers," Found. Trends Signal Process., vol. 12, nos. 3_4, pp. 200_431, 2018.

18. Y. Samir \& J. Shivajirao. (2020). Thermal infrared imaging based breast cancer diagnosis using machine learning techniques. Multimedia Tools and Applications. part of Springer Nature 2020 10.1007/s11042-020-09600-3.

19. S. Ekici \& H. Jawzal. Breast cancer diagnosis using thermography and convolutional neural networks. Medical Hypotheses. 2020 Apr; 137:109542. DOI: 10.1016/j.mehy.2019.109542.

20. H. Zadeh, A. Fayazi, B. Binazir, and M. Yargholi, (2020). "Breast Cancer Diagnosis Based on Feature Extraction Using Dynamic Models of Thermal Imaging and Deep Autoencoder Neural Networks," Journal of Testing and Evaluation 49. Published ahead of print, 07 August 2020, https://doi.org/10.1520/JTE20200044.

21. F.J. Fernández-Ovies, S. Alférez-Baquero, E.J. de Andrés-Galiana, A. Cernea, Z. Fernández-Muñiz, J.L. Fernández-Martínez (2019) Detection of Breast Cancer Using Infrared Thermography and Deep Neural Networks. In: Rojas I., Valenzuela O., Rojas F., Ortuño F. (eds) Bioinformatics and Biomedical Engineering. IWBBIO 2019. Lecture Notes in Computer Science, vol 11466. Springer, Cham. https://doi.org/10.1007/978-3-030-17935-9_46

22. M. Sebastien., O. Krejcar, P. Maresova, A. Selamat, \& K. Kuca. (2019) "Novel Four Stages Classification of Breast Cancer Using Infrared Thermal Imaging and a Deep Learning Model". In: Rojas I., Valenzuela O., Rojas F., Ortuño F. (eds) Bioinformatics and Biomedical Engineering. IWBBIO 2019. Lecture Notes in Computer Science, vol $11466 . \quad$ Springer, Cham. https://doi.org/10.1007/978-3-030-17935-9_7

23. J. Torres-Galván, E. Guevara and F. J. González, "Comparison of Deep Learning Architectures for Pre-Screening of Breast Cancer Thermograms," 2019 Photonics North (PN), Quebec City, QC,
Canada, 2019, pp. 1-2, doi: 10.1109/PN.2019.8819587.

24. Z. Juan Pablo \& A. Zeina \& B. Khaled \& M. Safa \& Z. Noureddine. (2020). A CNN-based methodology for breast cancer diagnosis using thermal images. Computer Methods in Biomechanics and Biomedical Engineering: Imaging \& Visualization. 1-15. 10.1080/21681163.2020.1824685.

25. H. Iqbal, B. Majeed, U. Khan, and M. A. Bin Altaf, "An Infrared High classification Accuracy Handheld Machine Learning based Breast-Cancer Detection System," 2019 IEEE Biomedical Circuits and Systems Conference (BioCAS), Nara, Japan, 2019, pp. 1-4, DOI: 10.1109/BIOCAS.2019.8918687.

26. M. Oliveira and L. Grassano "Convolutional Neural Networks for Static and Dynamic Breast Infrared Imaging Classification," 2018 31st SIBGRAPI Conference on Graphics, Patterns and Images (SIBGRAPI), Parana, Brazil, 2018, pp. 174-181, DOI: 10.1109/SIBGRAPI.2018.00029.

27. A. Dalmia, S.T. Kakileti, \& M. Geetha. (2018). Exploring Deep Learning Networks for Tumour Segmentation in Infrared Images. 10.21611/qirt.2018.052.

28. S. Mishra, A. Prakash, S. K. Roy, P. Sharan, and N. Mathur, "Breast Cancer Detection using Thermal Images and Deep Learning," 2020 7th International Conference on Computing for Sustainable Global Development (INDIACom), New Delhi, India, 2020, pp. 211-216, DOI: 10.23919/INDIACom49435.2020.9083722.

29. M. Farooq and P. Corcoran, "Infrared Imaging for Human Thermography and Breast Tumor Classification using Thermal Images," 2020 31st Irish Signals and Systems Conference (ISSC), Letterkenny, Ireland, 2020, pp. 1-6, DOI: 10.1109/ISSC49989.2020.9180164.

30. S. Tello-Mijares , F. Woo, and F. Flores. "Breast Cancer Identification via Thermography Image Segmentation with a Gradient Vector Flow and a Convolutional Neural Network". Journal of Healthcare Engineering. Volume 2019, Article ID 9807619, $13 \quad$ pages. https://doi.org/10.1155/2019/9807619

31. R. Roslidar, K. Saddami, F. Arnia, M. Syukri, and K. Munadi, "A Study of Fine-Tuning CNN Models Based on Thermal Imaging for Breast Cancer Classification," 2019 IEEE International Conference on Cybernetics and Computational Intelligence (CyberneticsCom), Banda Aceh, Indonesia, 2019, pp. $77-81$, DOI:

10.1109/CYBERNETICSCOM.2019.8875661. 